\title{
Upper Hölder continuity of parametric vector optimization problems
}

\author{
Xian-Fu Hu ${ }^{1 *}$ and Xiao-Wei Xue ${ }^{2}$
}

"Correspondence: xianfuhu@126.com

${ }^{1}$ School of Electrical Engineering, Chongqing City Management Vocational College, Chongqing, 401331, China

Full list of author information is available at the end of the article

\begin{abstract}
This paper is concerned with upper Hölder continuity and Hölder calmness of a perturbed vector optimization problem. We establish some new sufficient conditions for upper Hölder continuity and Hölder calmness of the perturbed solution mappings and the perturbed optimal value mappings of a vector optimization problem under the case that the objective function and the feasible set are, respectively, perturbed by parameters. Our results generalize and extend the corresponding ones of $\mathrm{Li}$ and $\mathrm{Li}$ (Appl. Math. Comput. 232:908-918, 2014).
\end{abstract}

MSC: 49K30; 90C29

Keywords: vector optimization problem; solution mapping; optimal value mapping; Hölder continuity; Hölder calmness

\section{Introduction}

The quantitative analysis of various optimal value mappings and optimal solution mappings for optimization problems is an interesting and important topic in optimization theories and applications. In general, it is understood as the optimal value mappings or optimal solution mappings possess some geometric properties, such as Hölder continuity, Lipschitz continuity, calmness, differentiability and sub-differentiability, and so on. It has implications in model formulation, optimality characterizations, approximation theory (especially for infinite dimensional problems), and in particular for numerical procedures. Thus, it is necessary to obtain some results for various optimal value mappings or various optimal solution mappings of optimization problems from a quantitative viewpoint. Up to now, there have been many papers to discuss Hölder continuity of solution mappings to perturbed variational inequalities or perturbed equilibrium problems; see [2-9] and the references therein.

However, there are a few papers to study this topic for perturbed optimization problems; see $[1,10,11]$ and the references therein. Bonnans and Shapiro [10] have used the growth conditions for real-valued mappings to consider a perturbed scalar optimization problem. They have derived upper Hölder continuity of the optimal solution mapping and the differentiability of the optimal value mapping. Bednarczuk [11] generalized the main results of [10] to the vector-valued mapping. Recently, Li and Li [1] introduced the strong convexity of the objective functions to derive Hölder continuity of the optimal solution mapping of a perturbed vector optimization problem. The main assumptions and proof

(c) Hu and Xue 2016. This article is distributed under the terms of the Creative Commons Attribution 4.0 International License (http://creativecommons.org/licenses/by/4.0/), which permits unrestricted use, distribution, and reproduction in any medium, provided you give appropriate credit to the original author(s) and the source, provide a link to the Creative Commons license, and indicate if changes were made. 
techniques are very different from $[10,11]$. However, there are many examples to show that the strong convexity of the objective functions of perturbed optimization problem is very strict. Thus, it is necessary to investigate Hölder continuity of the optimal solution mapping or the optimal value mapping for perturbed optimization problems.

In this paper, we introduce a new strong domination property, which is different from the corresponding ones in the related paper, for an unperturbed vector optimization problem. Based on this property and the Hölder-related assumptions, we establish the sufficient conditions for upper Hölder continuity and Hölder calmness of the various solution mappings and the optimal value mappings to a vector optimization problem under the case that the objective function and the feasible set are, respectively, perturbed by parameters. Comparing our results of this paper with the corresponding ones of [1], we can easily see that our results are not included in [1], and generalize and extend the corresponding ones of [1].

The rest of the paper is organized as follows. In Section 2, we first recall some definitions and their properties which are needed in the sequel. In Section 3, we discuss the upper Hölder continuity and Hölder calmness of the optimal solution mapping and the weak optimal solution mapping for a vector optimization problem. In Section 4, we further discuss the upper Hölder continuity of the optimal value mapping and the weak optimal value mapping for a vector optimization problem.

\section{Preliminaries}

Throughout this paper, we assume that $Y:=(Y,\|\cdot\|)$ is a normed space partially ordered by a point, closed and convex cone $C \subset Y$, which has a nonempty interior, denoted by int $C$. Let $D$ be a nonempty subset of $Y$. A point $y \in D$ is called an efficient point if $(D-y) \cap(-C)=$ $\{0\}$ and a weak efficient point if $(D-y) \cap(-\operatorname{int} C)=\emptyset$. We denote by $E(D)$ and $W E(D)$ the set of all efficient points and the set of all weak efficient points of $D$, respectively.

Let $X:=(X,\|\cdot\|), \Lambda:=(\Lambda,\|\cdot\|)$ and $M:=(M,\|\cdot\|)$ be normed spaces. We consider the perturbed vector optimization problem of form

$$
\left(\operatorname{VOP}_{\lambda, \mu}\right) \quad C-\min f(x, \lambda) \text { subject to } x \in \Phi(\mu) \text {, }
$$

depending on the parameters $\lambda \in \Lambda$ and $\mu \in M$, and $f: X \times \Lambda \rightarrow Y$ is a vector-valued mapping and $\Phi: M \rightarrow 2^{X}$ is a set-valued mapping with nonempty values.

For a given point $\left(\lambda_{0}, \mu_{0}\right) \in \Lambda \times M$, we view the corresponding problem $\left(\operatorname{VOP}_{\lambda_{0}, \mu_{0}}\right)$ as an unperturbed problem, and we identify the unperturbed problem $\left(\mathrm{VOP}_{\lambda_{0}, \mu_{0}}\right)$ with the problem (VOP) considered in the classical vector optimization theories and applications. That is, we use $f(\cdot) \equiv f\left(\cdot, \lambda_{0}\right)$ and $\Phi \equiv \Phi\left(\mu_{0}\right)$ and rewrite the unperturbed problem in the form

$$
\text { (VOP) } \quad C-\min f(x) \text { subject to } x \in \Phi \text {. }
$$

For any $(\lambda, \mu) \in \Lambda \times M$, the optimal value mapping and weak optimal value mapping for problem (2.1) are, respectively, defined by

$$
V(\lambda, \mu):=E(f(\Phi(\mu), \lambda)) \text { and } W V(\lambda, \mu):=W E(f(\Phi(\mu), \lambda))
$$


Then the solution mapping and weak solution mapping for problem (2.1) are, respectively, defined as

$$
\begin{aligned}
& S(\lambda, \mu):=\{x \in \in \Phi(\mu): f(x, \lambda) \in V(\lambda, \mu)\} \text { and } \\
& W S(\lambda, \mu):=\{x \in \in \Phi(\mu): f(x, \lambda) \in W V(\lambda, \mu)\} .
\end{aligned}
$$

For the convenience, we will use the abbreviated notations $V, W V, S$, and $W S$ to denote the optimal value mapping, the weak optimal value mapping, the solution mapping and the weak solution mapping for problem (2.2), respectively.

In this paper, we mainly study the behavior of the (weak) optimal value mapping and (weak) solution mapping for $(2.1)$ when $(\lambda, \mu)$ varies around the given point $\left(\lambda_{0}, \mu_{0}\right)$. Moreover, precisely speaking, we will discuss upper Hölder continuity of $V(\cdot), W V(\cdot), S(\cdot), W S(\cdot)$ as set-valued mappings at $(\lambda, \mu)$.

For any nonempty subsets $A, B$ of normed space, $d(x, B)=: \inf _{b \in B}\|x-b\|$ and $e(A, B)=$ $\sup _{a \in A} d(a, B)$. Let $G: M \rightrightarrows X$ be a set-valued mapping. The graph and domain of $G$ are, respectively, defined in the usual way as

$$
\operatorname{graph} G:=\{(\mu, x) \in M \times X \mid x \in G(\mu)\} \quad \text { and } \quad \operatorname{dom} G\{\mu \in M \mid G(\mu) \neq \emptyset\} .
$$

Definition 2.1 (i) $G$ is said to be Hölder of order $\alpha>0$ around $\mu_{0} \in \operatorname{dom} G$ with constants $k_{G}>0, t_{G}>0$ if and only if for any $\mu, \mu^{\prime} \in \mu_{0}+t_{G} \mathbb{B}_{M}$,

$$
e\left(G(\mu), G\left(\mu^{\prime}\right)\right) \leq k_{G}\left\|\mu-\mu^{\prime}\right\|^{\alpha}
$$

(ii) $G$ is said to be upper Hölder of order $\alpha>0$ around $\mu_{0} \in \operatorname{dom} G$ with constants $k_{G}>0, t_{G}>0$ if and only if for any $\mu \in \mu_{0}+t_{G} \mathbb{B}_{M}$,

$$
e\left(G(\mu), G\left(\mu_{0}\right)\right) \leq k_{G}\left\|\mu-\mu_{0}\right\|^{\alpha}
$$

(iii) $G$ is said to be lower Hölder of order $\alpha>0$ around $\mu_{0} \in \operatorname{dom} G$ with constants $k_{G}>0, t_{G}>0$ if and only if for any $\mu, \mu^{\prime} \in \mu_{0}+t_{G} \mathbb{B}_{M}$,

$$
e\left(G\left(\mu_{0}\right), G(\mu)\right) \leq k_{G}\left\|\mu-\mu_{0}\right\|^{\alpha}
$$

(iv) $G$ is said to be pseudo-Hölder of order $\alpha>0$ at $\left(\mu_{0}, x_{0}\right) \in \operatorname{graph} G$ with constants $k_{G}>0, t_{G}>0$ if and only if there exists a neighborhood $W$ of zero in $X$ such that, for any $\mu, \mu^{\prime} \in \mu_{0}+t_{G} \mathbb{B}_{M}$,

$$
e\left(G(\mu) \cap\left(x_{0}+W\right), G\left(\mu^{\prime}\right)\right) \leq k_{G}\left\|\mu-\mu^{\prime}\right\|^{\alpha} ;
$$

(v) $G$ is said to be Hölder calm or upper pseudo-Hölder of order $\alpha>0$ at $\left(\mu_{0}, x_{0}\right) \in \operatorname{graph} G$ with constants $k_{G}>0, t_{G}>0$ if and only if there exists a neighborhood $W$ of zero in $X$ such that, for any $\mu, \mu^{\prime} \in \mu_{0}+t_{G} \mathbb{B}_{M}$,

$$
e\left(G(\mu) \cap\left(x_{0}+W\right), G\left(\mu_{0}\right)\right) \leq k_{G}\left\|\mu-\mu_{0}\right\|^{\alpha} ;
$$


(vi) $G$ is said to be lower pseudo-Hölder of order $\alpha>0$ at $\left(\mu_{0}, x_{0}\right) \in \operatorname{graph} G$ with constants $k_{G}>0, t_{G}>0$ if and only if there exists a neighborhood $W$ of zero in $X$ such that, for any $\mu \in \mu_{0}+t_{G} \mathbb{B}_{M}$,

$$
e\left(G\left(\mu_{0}\right) \cap\left(x_{0}+W\right), G(\mu)\right) \leq k_{G}\left\|\mu-\mu_{0}\right\|^{\alpha} .
$$

From Definition 2.1, we have the following relations:

$$
\begin{aligned}
& \text { (i) } \Rightarrow(\text { ii }) \Rightarrow(v), \quad(\mathrm{i}) \Rightarrow(\text { iv }) \Rightarrow(v), \\
& (\text { i }) \Rightarrow(\text { iv }) \Rightarrow(\text { vi }), \quad(\mathrm{i}) \Rightarrow(\text { iii }) \Rightarrow(\text { vi }) .
\end{aligned}
$$

The set-valued mapping $G(\cdot)$ is Lipshitz (resp. upper Lipshitz, lower Lipshitz) around $\mu_{0} \in \operatorname{dom} G$ if $G(\cdot)$ is Hölder (resp. upper Hölder, lower Hölder) of order $\alpha=1$ around $\mu_{0} \in \operatorname{dom} G$; the set-valued mapping $G(\cdot)$ is pseudo-Lipshitz (resp. upper pseudo-Lipshitz, lower pseudo-Lipshitz) at $\left(\mu_{0}, x_{0}\right) \in$ graph $G$ if $G(\cdot)$ is pseudo-Hölder (resp. upper pseudoHölder, lower pseudo-Hölder) of order $\alpha=1$ at $\left(\mu_{0}, x_{0}\right) \in \operatorname{graph} G$. Pseudo-Lipshitzness and upper pseudo-Lipshishizness are also called the Aubin property and calmness, respectively. The role of Lipschitzness and its variants in optimization is widely recognized; see [10] and the references therein.

Definition 2.2 A vector-valued mapping $g: M \rightarrow X$ is Hölder of order $\alpha>0$ around $\mu_{0} \in$ $M$ with constants $k_{g}, t_{g}>0$ if and only if for any $\mu, \mu^{\prime} \in \mu_{0}+t_{g} \mathbb{B}_{M}$,

$$
\left\|g(\mu)-g\left(\mu^{\prime}\right)\right\| \leq k_{g}\left\|\mu-\mu^{\prime}\right\|^{\alpha} .
$$

Let $D$ be a subset of $Y$. Luc [12] introduced the domination property (for short, DP) for D:

$$
D \subset E(D)+C
$$

Then the domination property (DP) for problem (VOP) can be similarly defined by

$$
f(\Phi) \subset f(S)+C
$$

that is, for each $x \in \Phi$, there exists $x_{0} \in S$ such that

$$
f(x)-f\left(x_{0}\right) \in C
$$

Motivated by the above property, we introduce the following concepts for problem (VOP) to discuss Hölder continuity of the perturbed solution mapping and perturbed optimal value mapping of $\left(\operatorname{VOP}_{\lambda, \mu}\right)$.

Definition 2.3 (i) The strong global domination property (for short, SGDP) holds on $S$ (resp. WS) of order $\alpha>0$ with constant $h_{s}>0$ for (VOP) if and only if for each $x \in \Phi$, there exists $x_{0} \in S$ (resp. WS) such that

$$
f(x)-f\left(x_{0}\right)+h_{s}\left\|x-x_{0}\right\|^{\alpha} \mathbb{B}_{Y} \subset C .
$$


(ii) The strong local domination property (for short, SLDP) holds around $x_{0} \in W$ (resp. $W S$ ) of order $\alpha>0$ with constant $h_{s}>0$ for (VOP) if and only if there exists a neighborhood $U$ of $x_{0}$ such that, for each $x \in \Phi \cap U$, there exists $x_{0} \in S \cap U$ (resp. WS $\cap U$ ) satisfies (2.3).

Remark 2.1 (i) For any $y \in Y$, let $\|y\|_{+}:=d(y, Y \backslash C)$. Then equation (2.3) becomes

$$
h_{s}\left\|x-x_{0}\right\|^{\alpha} \leq\left\|f(x)-f\left(x_{0}\right)\right\|_{+} .
$$

(ii) The concepts of Definition 2.3 are mainly to establish Hölder-type continuity of problem (VOP), which was discussed by Li and Li [1]. In [1], they mainly introduced the strong convexity of the objective function in (VOP) to investigate this topic. However, the assumption on strong convexity of the objective function is very strict. In many cases, the strong global (local) domination property for (VOP) holds but the strong convexity of the objective function fails as the following example shows.

Example 2.1 Let $X=\mathbb{R}, Y=\mathbb{R}^{2}, C=\mathbb{R}_{+}^{2}, \Phi=[-1,1]$, and $f: X \rightarrow Y$ is $f(x)=(x, x)$. We can easy see that $f$ is not strongly convex on $\Phi$; the solution sets $S=W S=[-1,0]$. Hence, for any $x \in \Phi$, there exist a constant $h_{s}=\frac{\sqrt{2}}{2}$ and $x_{0}=0 \in S=W S$ such that

$$
f(x)-f\left(x_{0}\right)+\frac{\sqrt{2}}{2}\left\|x-x_{0}\right\| \mathbb{B}_{Y} \subset C .
$$

That is, the strong global domination property for (VOP) on $S$ (resp. WS) holds.

\section{Upper Hölder continuity of the solution mappings for $\left(\mathrm{VOP}_{\lambda, \mu}\right)$}

In this section, we mainly discuss upper Hölder behavior of the perturbed (weak) solution mapping for problem (2.1) where both the objective function $f$ and the feasible set $\Phi$ are perturbed by different parameters. Namely, this section is devoted to derive the upper bounds for the distance from the perturbed (weak) solution mapping of (2.1) to the (weak) solution mapping for problem (2.2).

Theorem 3.1 For problem (2.1), assume that the solutions exist in a neighborhood of considered point $\left(\lambda_{0}, \mu_{0}\right) \in \operatorname{dom} S$. Moreover, assume that the following conditions are satisfied:

(i) the set-valued mapping $\Phi: M \rightrightarrows X$ is Hölder of order $\alpha>0$ around $\mu_{0} \in \operatorname{dom} \Phi$ with constants $h_{\Phi}>0, t_{\Phi}>0$;

(ii) the strong global domination property (SGDP) of order $\beta \geq 1$ for (VOP) holds on $S$ with a constant $l_{s}>0$;

(iii) for any $\lambda \in \Lambda, f(\cdot, \lambda)$ is Hölder of order $\gamma>0$ on $X$ with constant $m_{f}>0$ as well as for any $x \in X, f(x, \cdot)$ is Hölder of order $\delta>0$ around $\lambda_{0}$ with constants $n_{f}>0, t_{f}>0$.

Then $S: \Lambda \times M \rightrightarrows X$ is upper Hölder around $\left(\lambda_{0}, \mu_{0}\right) \in \operatorname{dom} S$ with constants $t_{f}>0, t_{\Phi}>0$, that is, for any $\lambda \in \lambda_{0}+t_{f} \mathbb{B}_{\Lambda}, \mu \in \mu_{0}+t_{\Phi} \mathbb{B}_{M}$,

$$
e(S(\lambda, \mu), S) \leq h_{\Phi}\left\|\mu-\mu_{0}\right\|^{\alpha}+\left(\frac{2 m_{f} h_{\Phi}^{\gamma}}{l_{s}}\right)^{\frac{1}{\beta}}\left\|\mu-\mu_{0}\right\|^{\frac{\alpha \gamma}{\beta}}+\left(\frac{2 n_{f}}{l_{s}}\right)^{\frac{1}{\beta}}\left\|\lambda-\lambda_{0}\right\|^{\frac{\delta}{\beta}} .
$$

Proof Take any $x(\lambda, \mu) \in S(\lambda, \mu)$, for any $\lambda \in \lambda_{0}+t_{f} \mathbb{B}_{\Lambda}, \mu \in \mu_{0}+t_{\Phi} \mathbb{B}_{M}$. By the definition of $S(\lambda, \mu)$, we have

$$
x(\lambda, \mu) \in \Phi(\mu) \quad \text { and } \quad f(x, \lambda)-f(x(\lambda, \mu), \lambda) \notin-C \backslash\{0\} \quad \text { for any } x \in \Phi(\mu) .
$$


Then it follows from (i) that there exists $x\left(\mu_{0}\right) \in \Phi\left(\mu_{0}\right)$ such that

$$
\left\|x(\lambda, \mu)-x\left(\mu_{0}\right)\right\| \leq h_{\Phi}\left\|\mu-\mu_{0}\right\|^{\alpha} .
$$

By the strong domination property, for $x\left(\mu_{0}\right)$, there exists $x\left(\lambda_{0}, \mu_{0}\right) \in S$ such that

$$
f\left(x\left(\mu_{0}\right), \lambda_{0}\right)-f\left(x\left(\lambda_{0}, \mu_{0}\right), \lambda_{0}\right)+l_{s}\left\|x\left(\mu_{0}\right)-x\left(\lambda_{0}, \mu_{0}\right)\right\|^{\beta} \mathbb{B}_{Y} \subset C .
$$

The conclusion (3.1) is trivial whether $x\left(\mu_{0}\right)=x\left(\lambda_{0}, \mu_{0}\right)$ or $f\left(x\left(\mu_{0}\right), \lambda_{0}\right)=f\left(x\left(\lambda_{0}, \mu_{0}\right), \lambda_{0}\right)$. Therefore, $x\left(\mu_{0}\right) \neq x\left(\lambda_{0}, \mu_{0}\right)$ and $f\left(x\left(\mu_{0}\right), \lambda_{0}\right) \neq f\left(x\left(\lambda_{0}, \mu_{0}\right), \lambda_{0}\right)$ are always assumed in the sequel. Clearly, $x\left(\lambda_{0}, \mu_{0}\right) \in \Phi\left(\mu_{0}\right)$. By (i), there exists $x(\mu) \in \Phi(\mu)$ such that

$$
\left\|x\left(\lambda_{0}, \mu_{0}\right)-x(\mu)\right\| \leq h_{\Phi}\left\|\mu-\mu_{0}\right\|^{\alpha} .
$$

Then we have

$$
f(x(\mu), \lambda)-f(x(\lambda, \mu), \lambda)=f\left(x\left(\lambda_{0}, \mu_{0}\right), \lambda_{0}\right)-f\left(x\left(\mu_{0}\right), \lambda_{0}\right)+w,
$$

where

$$
\begin{aligned}
w:= & {\left[f(x(\mu), \lambda)-f\left(x\left(\lambda_{0}, \mu_{0}\right), \lambda\right)\right]+\left[f\left(x\left(\lambda_{0}, \mu_{0}\right), \lambda\right)-f\left(x\left(\lambda_{0}, \mu_{0}\right), \lambda_{0}\right)\right] } \\
& +\left[f\left(x\left(\mu_{0}\right), \lambda_{0}\right)-f\left(x\left(\mu_{0}\right), \lambda\right)\right]+\left[f\left(x\left(\mu_{0}\right), \lambda\right)-f(x(\lambda, \mu), \lambda)\right] .
\end{aligned}
$$

Then it follows from (iii) that

$$
\|w\| \leq m_{f}\left\|x(\mu)-x\left(\lambda_{0}, \mu_{0}\right)\right\|^{\gamma}+m_{f}\left\|x\left(\mu_{0}\right)-x(\lambda, \mu)\right\|^{\gamma}+2 n_{f}\left\|\lambda-\lambda_{0}\right\|^{\delta},
$$

which together with (3.3) and (3.5) yields

$$
\|w\| \leq 2 m_{f} h_{\Phi}^{\gamma}\left\|\mu-\mu_{0}\right\|^{\alpha \gamma}+2 n_{f}\left\|\lambda-\lambda_{0}\right\|^{\delta} .
$$

We claim that

$$
\left\|f\left(x\left(\mu_{0}\right), \lambda_{0}\right)-f\left(x\left(\lambda_{0}, \mu_{0}\right), \lambda_{0}\right)\right\|_{+} \leq\|w\| .
$$

Indeed, suppose to the contrary that $\left\|f\left(x\left(\mu_{0}\right), \lambda_{0}\right)-f\left(x\left(\lambda_{0}, \mu_{0}\right), \lambda_{0}\right)\right\|_{+}>\|w\|$. Then, by the definition of $\|\cdot\|_{+}$,

$$
f\left(x\left(\mu_{0}\right), \lambda_{0}\right)-f\left(x\left(\lambda_{0}, \mu_{0}\right), \lambda_{0}\right)+\|w\| \mathbb{B}_{Y} \subset \operatorname{int} C .
$$

If $\|w\|=0$, then $w=0$. By (3.4) and (3.6), we have

$$
f(x(\mu), \lambda)-f(x(\lambda, \mu), \lambda) \in-\operatorname{int} C,
$$

which contradicts (3.2). If $\|w\| \neq 0$, then, by $-\frac{w}{\|w\|} \in \mathbb{B}_{Y}$ and (3.9),

$$
f(x(\lambda, \mu), \lambda)-f(x(\mu), \lambda)=f\left(x\left(\mu_{0}\right), \lambda_{0}\right)-f\left(x\left(\lambda_{0}, \mu_{0}\right), \lambda_{0}\right)+\|w\|\left(-\frac{w}{\|w\|}\right) \in \operatorname{int} C,
$$

which also contradicts (3.2). 
Therefore, combining (3.4), (3.7), (3.8), and (3.9), we have

$$
\begin{aligned}
l_{s}\left\|x\left(\mu_{0}\right)-x\left(\lambda_{0}, \mu_{0}\right)\right\|^{\beta} & \leq\left\|f\left(x\left(\mu_{0}\right), \lambda_{0}\right)-f\left(x\left(\lambda_{0}, \mu_{0}\right), \lambda_{0}\right)\right\|_{+} \\
& \leq 2 m_{f} h_{\Phi}^{\gamma}\left\|\mu-\mu_{0}\right\|^{\alpha \gamma}+2 n_{f}\left\|\lambda-\lambda_{0}\right\|^{\delta} .
\end{aligned}
$$

So

$$
\begin{aligned}
\left\|x\left(\mu_{0}\right)-x\left(\lambda_{0}, \mu_{0}\right)\right\| & \leq \sqrt[\beta]{\frac{2 m_{f} h_{\Phi}^{\gamma}\left\|\mu-\mu_{0}\right\|^{\alpha \gamma}}{l_{s}}\left\|m u-\mu_{0}\right\|^{\alpha \gamma}+\frac{2 n_{f}}{l_{s}}\left\|\lambda-\lambda_{0}\right\|^{\delta}} \\
& \leq\left(\frac{2 m_{f} h_{\Phi}^{\gamma}}{l_{s}}\right)^{\frac{1}{\beta}}\left\|\mu-\mu_{0}\right\|^{\frac{\alpha \gamma}{\beta}}+\left(\frac{2 n_{f}}{l_{s}}\right)^{\frac{1}{\beta}}\left\|\lambda-\lambda_{0}\right\|^{\frac{\delta}{\beta}},
\end{aligned}
$$

where the last inequality holds as $\beta \geq 1$.

Finally, from (3.2) and (3.10) it follows that

$$
\begin{aligned}
d(x(\lambda, \mu), S) & \leq\left\|x(\lambda, \mu)-x\left(\lambda_{0}, \mu_{0}\right)\right\| \leq\left\|x(\lambda, \mu)-x\left(\mu_{0}\right)\right\|+\left\|x\left(\mu_{0}\right)-x\left(\lambda_{0}, \mu_{0}\right)\right\| \\
& \leq h_{\Phi}\left\|\mu-\mu_{0}\right\|^{\alpha}+\left(\frac{2 m_{f} h_{\Phi}^{\gamma}}{l_{s}}\right)^{\frac{1}{\beta}}\left\|\mu-\mu_{0}\right\|^{\frac{\alpha \gamma}{\beta}}+\left(\frac{2 n_{f}}{l_{s}}\right)^{\frac{1}{\beta}}\left\|\lambda-\lambda_{0}\right\|^{\frac{\delta}{\beta}},
\end{aligned}
$$

which means that the conclusion (3.1) holds and the proof is complete.

Theorem 3.2 For problem (2.1), assume that the weak solutions exist in a neighborhood of considered point $\left(\lambda_{0}, \mu_{0}\right) \in$ dom WS. Furthermore, assume that all conditions of Theorem 3.1 are satisfied except that (ii) is replaced by

(ii') the strong global domination property (SGDP) of order $\beta \geq 1$ for (VOP) holds on WS with a constant $l_{s}>0$.

Then WS $: \Lambda \times M \rightrightarrows X$ is upper Hölder around $\left(\lambda_{0}, \mu_{0}\right) \in$ dom WS with constants $t_{f}>0$, $t_{\Phi}>0$, that is, for any $\lambda \in \lambda_{0}+t_{f} \mathbb{B}_{\Lambda}, \mu \in \mu_{0}+t_{\Phi} \mathbb{B}_{M}$,

$$
e(W S(\lambda, \mu), W S) \leq h_{\Phi}\left\|\mu-\mu_{0}\right\|^{\alpha}+\left(\frac{2 m_{f} h_{\Phi}^{\gamma}}{l_{s}}\right)^{\frac{1}{\beta}}\left\|\mu-\mu_{0}\right\|^{\frac{\alpha \gamma}{\beta}}+\left(\frac{2 n_{f}}{l_{s}}\right)^{\frac{1}{\beta}}\left\|\lambda-\lambda_{0}\right\|^{\frac{\delta}{\beta}} .
$$

Proof The proof is similar to Theorem 3.1 and is omitted here.

Theorem 3.3 For problem (2.1), assume that the solutions exist in a neighborhood of considered point $\left(\lambda_{0}, \mu_{0}\right) \in \operatorname{dom} S$. Let $x_{0} \in S$. Furthermore, assume that the following conditions are satisfied:

(i) the set-valued mapping $\Phi: M \rightrightarrows X$ is Hölder calm and lower pseudo-Hölder of order $\alpha>0$ around $\left(\mu_{0}, x_{0}\right) \in$ graph $\Phi$ with a neighborhood $V$ of zero in $X$, and constants $h_{\Phi}>0, c_{\Phi}>0$;

(ii) the strong local domination property (SLDP) of order $\beta \geq 1$ holds for (VOP) around $x_{0}$ with a neighborhood $Q$ of zero in $X, Q+Q \subset V$, and a constant $l_{s}>0$;

(iii) for any $x \in x_{0}+V, f(x, \cdot)$ is Hölder of order $\delta>0$ around $\lambda_{0}$ with constants $n_{f}>0$, $t_{f}>0$ as well as for any $\lambda \in \lambda_{0}+t_{f} \mathbb{B}_{\Lambda}, f(\cdot, \lambda)$ is Hölder of order $\gamma>0$ with a neighborhood $V$ of zero in $X$, and a constant $m_{f}>0$. 
Then $S: \Lambda \times M \rightrightarrows X$ is Hölder calm around $\left(\left(\lambda_{0}, \mu_{0}\right), x_{0}\right) \in \operatorname{graph} S$ with constants $t_{f}>0$, $t_{\Phi}>0$. Precisely, there exists a neighborhood $W$ of zero in $X$ such that for any $\lambda \in \lambda_{0}+t_{f} \mathbb{B}_{\Lambda}$, $\mu \in \mu_{0}+t_{\Phi} \mathbb{B}_{M}$,

$$
\begin{aligned}
& e\left(S(\lambda, \mu) \cap\left(x_{0}+W\right), S\right) \\
& \quad \leq h_{\Phi}\left\|\mu-\mu_{0}\right\|^{\alpha}+\left(\frac{2 m_{f} h_{\Phi}^{\gamma}}{l_{s}}\right)^{\frac{1}{\beta}}\left\|\mu-\mu_{0}\right\|^{\frac{\alpha \gamma}{\beta}}+\left(\frac{2 n_{f}}{l_{s}}\right)^{\frac{1}{\beta}}\left\|\lambda-\lambda_{0}\right\|^{\frac{\delta}{\beta}} .
\end{aligned}
$$

Proof Let $W$ be any neighborhood of zero in $X$ such that

$$
W+h_{\Phi} t_{\Phi} \mathbb{B}_{X} \subset Q
$$

Then $W$ is the desired neighborhood of zero in $X$. Indeed, take any $x(\lambda, \mu) \in S(\lambda, \mu) \cap$ $\left(x_{0}+W\right)$, for any $\lambda \in \lambda_{0}+t_{f} \mathbb{B}_{\Lambda}, \mu \in \mu_{0}+t_{\Phi} \mathbb{B}_{M}$. By the Hölder calmness of $\Phi$, there exists $x\left(\mu_{0}\right) \in \Phi\left(\mu_{0}\right)$ such that

$$
\left\|x(\lambda, \mu)-x\left(\mu_{0}\right)\right\| \leq h_{\Phi}\left\|\mu-\mu_{0}\right\|^{\alpha} \leq h_{\Phi} t_{\Phi}^{\alpha} .
$$

Then we have

$$
x\left(\mu_{0}\right)-x_{0}=x\left(\mu_{0}\right)-x(\lambda, \mu)+x(\lambda, \mu)-x_{0} \in h_{\Phi} t_{\Phi}^{\alpha} \mathbb{B}_{X}+W \subset Q .
$$

For $x\left(\mu_{0}\right) \in \Phi\left(\mu_{0}\right) \cap\left(x_{0}+Q\right)$, by (ii), there exists $x\left(\lambda_{0}, \mu_{0}\right) \in S \cap\left(x_{0}+Q\right)$ such that

$$
f\left(x\left(\mu_{0}\right), \lambda_{0}\right)-f\left(x\left(\lambda_{0}, \mu_{0}\right), \lambda_{0}\right)+l\left\|x\left(\mu_{0}\right)-x\left(\lambda_{0}, \mu_{0}\right)\right\|^{\beta} \mathbb{B}_{Y} \subset C .
$$

Without loss of generality, we assume that $x\left(\mu_{0}\right) \neq x\left(\lambda_{0}, \mu_{0}\right)$ and $f\left(x\left(\mu_{0}\right), \lambda_{0}\right) \neq f\left(x\left(\lambda_{0}, \mu_{0}\right)\right.$, $\left.\lambda_{0}\right)$. Otherwise, the conclusion (3.11) is trivial. Obviously, $x\left(\lambda_{0}, \mu_{0}\right) \in \Phi\left(\mu_{0}\right) \cap\left(x_{0}+Q\right)$. By the lower-pseudo Hölder property of $\Phi$, there exists $x(\mu) \in \Phi(\mu)$ such that

$$
\left\|x\left(\lambda_{0}, \mu_{0}\right)-x(\mu)\right\| \leq h_{\Phi}\left\|\mu-\mu_{0}\right\|^{\alpha} \leq h_{\Phi} t_{\Phi}^{\alpha} .
$$

So, we have

$$
x\left(\mu_{0}\right)-x_{0}=x\left(\mu_{0}\right)-x\left(\lambda_{0}, \mu_{0}\right)+x\left(\lambda_{0}, \mu_{0}\right)-x_{0} \in h_{\Phi} t_{\Phi}^{\alpha} \mathbb{B}_{X}+W \subset Q \subset V
$$

and

$$
f(x(\mu), \lambda)-f(x(\lambda, \mu), \lambda)=f\left(x\left(\lambda_{0}, \mu_{0}\right), \lambda_{0}\right)-f\left(x\left(\mu_{0}\right), \lambda_{0}\right)+w,
$$

where

$$
\begin{aligned}
w:= & {\left[f(x(\mu), \lambda)-f\left(x\left(\lambda_{0}, \mu_{0}\right), \lambda\right)\right]+\left[f\left(x\left(\lambda_{0}, \mu_{0}\right), \lambda\right)-f\left(x\left(\lambda_{0}, \mu_{0}\right), \lambda_{0}\right)\right] } \\
& +\left[f\left(x\left(\mu_{0}\right), \lambda_{0}\right)-f\left(x\left(\mu_{0}\right), \lambda\right)\right]+\left[f\left(x\left(\mu_{0}\right), \lambda\right)-f(x(\lambda, \mu), \lambda)\right] .
\end{aligned}
$$


Then, by (iii), we have

$$
\|w\| \leq m_{f}\left\|x(\mu)-x\left(\lambda_{0}, \mu_{0}\right)\right\|^{\gamma}+m_{f}\left\|x\left(\mu_{0}\right)-x(\lambda, \mu)\right\|^{\gamma}+2 n_{f}\left\|\lambda-\lambda_{0}\right\|^{\delta},
$$

which together with (3.12) and (3.14) yields

$$
\|w\| \leq 2 m_{f} h_{\Phi}^{\gamma}\left\|\mu-\mu_{0}\right\|^{\alpha \gamma}+2 n_{f}\left\|\lambda-\lambda_{0}\right\|^{\delta} .
$$

By the same arguments as in the proof of Theorem 3.1, we have

$$
\left\|f\left(x\left(\mu_{0}\right), \lambda_{0}\right)-f\left(x\left(\lambda_{0}, \mu_{0}\right), \lambda_{0}\right)\right\|_{+} \leq\|w\| .
$$

Therefore, (3.13), (3.15), and (3.16) together yield

$$
l_{s}\left\|x\left(\mu_{0}\right)-x\left(\lambda_{0}, \mu_{0}\right)\right\|^{\beta} \leq 2 m_{f} h_{\Phi}^{\gamma}\left\|\mu-\mu_{0}\right\|^{\alpha \gamma}+2 n_{f}\left\|\lambda-\lambda_{0}\right\|^{\delta} .
$$

So, we have

$$
\left\|x\left(\mu_{0}\right)-x\left(\lambda_{0}, \mu_{0}\right)\right\| \leq\left(\frac{2 m_{f} h_{\Phi}^{\gamma}}{l_{s}}\right)^{\frac{1}{\beta}}\left\|\mu-\mu_{0}\right\|^{\frac{\alpha \gamma}{\beta}}+\left(\frac{2 n_{f}}{l_{s}}\right)^{\frac{1}{\beta}}\left\|\lambda-\lambda_{0}\right\|^{\frac{\delta}{\beta}}
$$

Finally, from (3.12) and (3.17) it follows that

$$
d(x(\lambda, \mu), S) \leq h_{\Phi}\left\|\mu-\mu_{0}\right\|^{\alpha}+\left(\frac{2 m_{f} h_{\Phi}^{\gamma}}{l_{s}}\right)^{\frac{1}{\beta}}\left\|\mu-\mu_{0}\right\|^{\frac{\alpha \gamma}{\beta}}+\left(\frac{2 n_{f}}{l_{s}}\right)^{\frac{1}{\beta}}\left\|\lambda-\lambda_{0}\right\|^{\frac{\delta}{\beta}}
$$

which means that the conclusion (3.11) holds and the proof is complete.

Theorem 3.4 For problem (2.1), assume that the weak solutions exist in a neighborhood of considered point $\left(\lambda_{0}, \mu_{0}\right) \in \operatorname{dom}$ WS. Let $x_{0} \in$ WS. Furthermore, assume that the following conditions of Theorem 3.2 are satisfied. Then WS : $\Lambda \times M \rightrightarrows X$ is Hölder calm around $\left(\left(\lambda_{0}, \mu_{0}\right), x_{0}\right) \in$ graph WS with constants $t_{f}>0, t_{\Phi}>0$, that is, there exists a neighborhood $W$ of zero in $X$ such that for any $\lambda \in \lambda_{0}+t_{f} \mathbb{B}_{\Lambda}, \mu \in \mu_{0}+t_{\Phi} \mathbb{B}_{M}$,

$$
\begin{aligned}
& e\left(W S(\lambda, \mu) \cap\left(x_{0}+W\right), W S\right) \\
& \quad \leq h_{\Phi}\left\|\mu-\mu_{0}\right\|^{\alpha}+\left(\frac{2 m_{f} h_{\Phi}^{\gamma}}{l_{s}}\right)^{\frac{1}{\beta}}\left\|\mu-\mu_{0}\right\|^{\frac{\alpha \gamma}{\beta}}+\left(\frac{2 n_{f}}{l_{s}}\right)^{\frac{1}{\beta}}\left\|\lambda-\lambda_{0}\right\|^{\frac{\delta}{\beta}} .
\end{aligned}
$$

Proof The proof is similar to Theorem 3.3 and is omitted here.

\section{Upper Hölder of the optimal value mappings for $\left(\operatorname{VOP}_{\lambda, \mu}\right)$}

This section is devoted to an investigation of the Hölder behavior of the perturbed optimal value mappings for problem (2.1).

Theorem 4.1 Assume that all conditions of Theorem 3.1 are satisfied. Moreover, assume that $\gamma=1$ in Theorem 3.1. Then $V: \Lambda \times M \rightrightarrows X$ is upper Hölder around $\left(\lambda_{0}, \mu_{0}\right) \in \operatorname{dom} S$ 
with constants $t_{f}>0, t_{\Phi}>0$. Precisely, for any $\lambda \in \lambda_{0}+t_{f} \mathbb{B}_{\Lambda}, \mu \in \mu_{0}+t_{\Phi} \mathbb{B}_{M}$,

$$
\begin{aligned}
e(V(\lambda, \mu), V) \leq & m_{f} h_{\Phi}\left\|\mu-\mu_{0}\right\|^{\alpha}+n_{f}\left\|\lambda-\lambda_{0}\right\|^{\delta}+\left(\frac{2 m_{f}^{2} h_{\Phi}}{l_{s}}\right)^{\frac{1}{\beta}}\left\|\mu-\mu_{0}\right\|^{\frac{\alpha}{\beta}} \\
& +\left(\frac{2 m_{f} n_{f}}{l_{s}}\right)^{\frac{1}{\beta}}\left\|\lambda-\lambda_{0}\right\|^{\frac{\delta}{\beta}} .
\end{aligned}
$$

Moreover, if $\beta=1$, then

$$
e(V(\lambda, \mu), V) \leq \frac{\left(2 m_{f}+l_{s}\right) m_{f} h_{\Phi}}{l_{s}}\left\|\mu-\mu_{0}\right\|^{\alpha}+\frac{\left(2 m_{f}+l_{s}\right) n_{f}}{l_{s}}\left\|\lambda-\lambda_{0}\right\|^{\delta} .
$$

Proof Take any $f(x(\lambda, \mu), \lambda) \in V(\lambda, \mu)$, for any $\lambda \in \lambda_{0}+t_{f} \mathbb{B}_{\Lambda}, \mu \in \mu_{0}+t_{\Phi} \mathbb{B}_{M}$. Then $x(\lambda, \mu) \in$ $\Phi(\mu)$ and there exists $x\left(\mu_{0}\right) \in \Phi\left(\mu_{0}\right)$ such that

$$
\left\|x(\lambda, \mu)-x\left(\mu_{0}\right)\right\| \leq h_{\Phi}\left\|\mu-\mu_{0}\right\|^{\alpha} .
$$

Then it follows from (ii) that, for $x\left(\mu_{0}\right)$, there exists $x\left(\lambda_{0}, \mu_{0}\right) \in S$ such that

$$
f\left(x\left(\mu_{0}\right), \lambda_{0}\right)-f\left(x\left(\lambda_{0}, \mu_{0}\right), \lambda_{0}\right)+l_{s}\left\|x\left(\mu_{0}\right)-x\left(\lambda_{0}, \mu_{0}\right)\right\|^{\beta} \mathbb{B}_{Y} \subset C .
$$

So, the Lipchitzness of $f\left(\cdot, \lambda_{0}\right)$ implies that

$$
f\left(x\left(\mu_{0}\right), \lambda_{0}\right)-f\left(x\left(\lambda_{0}, \mu_{0}\right), \lambda_{0}\right)+\frac{l_{s}}{m_{f}}\left\|f\left(x\left(\mu_{0}\right), \lambda_{0}\right)-f\left(x\left(\lambda_{0}, \mu_{0}\right), \lambda_{0}\right)\right\|^{\beta} \mathbb{B}_{Y} \subset C .
$$

Clearly, $x\left(\lambda_{0}, \mu_{0}\right) \in \Phi\left(\mu_{0}\right)$. Then, by (i), there exists $x(\mu) \in \Phi(\mu)$ such that

$$
\left\|x\left(\lambda_{0}, \mu_{0}\right)-x(\mu)\right\| \leq h_{\Phi}\left\|\mu-\mu_{0}\right\|^{\alpha}
$$

Obviously, we have

$$
f(x(\mu), \lambda)-f(x(\lambda, \mu), \lambda)=f\left(x\left(\lambda_{0}, \mu_{0}\right), \lambda_{0}\right)-f\left(x\left(\mu_{0}\right), \lambda_{0}\right)+w,
$$

where

$$
\begin{aligned}
w:= & {\left[f(x(\mu), \lambda)-f\left(x\left(\lambda_{0}, \mu_{0}\right), \lambda\right)\right]+\left[f\left(x\left(\lambda_{0}, \mu_{0}\right), \lambda\right)-f\left(x\left(\lambda_{0}, \mu_{0}\right), \lambda_{0}\right)\right] } \\
& +\left[f\left(x\left(\mu_{0}\right), \lambda_{0}\right)-f\left(x\left(\mu_{0}\right), \lambda\right)\right]+\left[f\left(x\left(\mu_{0}\right), \lambda\right)-f(x(\lambda, \mu), \lambda)\right] .
\end{aligned}
$$

Then it follows from (iii) that

$$
\|w\| \leq m_{f}\left\|x(\mu)-x\left(\lambda_{0}, \mu_{0}\right)\right\|+m_{f}\left\|x\left(\mu_{0}\right)-x(\lambda, \mu)\right\|+2 n_{f}\left\|\lambda-\lambda_{0}\right\|^{\delta},
$$

which together with (4.1) and (4.3) yields

$$
\|w\| \leq 2 m_{f} h_{\Phi}\left\|\mu-\mu_{0}\right\|^{\alpha}+2 n_{f}\left\|\lambda-\lambda_{0}\right\|^{\delta} .
$$


By the same arguments as in the proof of Theorem 3.1, we have

$$
\left\|f\left(x\left(\mu_{0}\right), \lambda_{0}\right)-f\left(x\left(\lambda_{0}, \mu_{0}\right), \lambda_{0}\right)\right\|_{+} \leq\|w\|
$$

Thus, (4.2), (4.4), and (4.5) together yield

$$
\frac{l_{s}}{m_{f}}\left\|f\left(x\left(\mu_{0}\right), \lambda_{0}\right)-f\left(x\left(\lambda_{0}, \mu_{0}\right), \lambda_{0}\right)\right\|^{\beta} \leq 2 m_{f} h_{\Phi}\left\|\mu-\mu_{0}\right\|^{\alpha}+2 n_{f}\left\|\lambda-\lambda_{0}\right\|^{\delta} .
$$

So, we have

$$
\begin{aligned}
& \left\|f\left(x\left(\mu_{0}\right), \lambda_{0}\right)-f\left(x\left(\lambda_{0}, \mu_{0}\right), \lambda_{0}\right)\right\| \\
& \quad \leq\left(\frac{2 m_{f}^{2} h_{\Phi}}{l_{s}}\right)^{\frac{1}{\beta}}\left\|\mu-\mu_{0}\right\|^{\frac{\alpha}{\beta}}+\left(\frac{2 m_{f} n_{f}}{l_{s}}\right)^{\frac{1}{\beta}}\left\|\lambda-\lambda_{0}\right\|^{\frac{\delta}{\beta}} .
\end{aligned}
$$

Finally, from (4.1) and (4.6) it follows that

$$
\begin{aligned}
d( & f(x(\lambda, \mu), \lambda), V) \\
\leq & \left\|f(x(\lambda, \mu), \lambda)-f\left(x\left(\lambda_{0}, \mu_{0}\right), \lambda_{0}\right)\right\| \\
\leq & \left\|f(x(\lambda, \mu), \lambda)-f\left(x\left(\mu_{0}\right), \lambda\right)\right\|+\left\|f\left(x\left(\mu_{0}\right), \lambda\right)-f\left(x\left(\lambda_{0}, \mu_{0}\right), \lambda_{0}\right)\right\| \\
\quad & +\left\|f\left(x\left(\mu_{0}\right), \lambda_{0}\right)-f\left(x\left(\lambda_{0}, \mu_{0}\right), \lambda_{0}\right)\right\| \\
\leq & m_{f} h_{\Phi}\left\|\mu-\mu_{0}\right\|^{\alpha}+n_{f}\left\|\lambda-\lambda_{0}\right\|^{\delta}+\left(\frac{2 m_{f}^{2} h_{\Phi}}{l_{s}}\right)^{\frac{1}{\beta}}\left\|\mu-\mu_{0}\right\|^{\frac{\alpha}{\beta}} \\
& +\left(\frac{2 m_{f} n_{f}}{l_{s}}\right)^{\frac{1}{\beta}}\left\|\lambda-\lambda_{0}\right\|^{\frac{\delta}{\beta}} .
\end{aligned}
$$

The proof is complete.

Theorem 4.2 Assume that all conditions of Theorem 3.2 are satisfied. Moreover, assume that $\gamma=1$ in Theorem 3.2. Then $W V: \Lambda \times M \rightrightarrows X$ is upper Hölder around $\left(\lambda_{0}, \mu_{0}\right) \in$ dom WS with constants $t_{f}>0, t_{\Phi}>0$. Precisely, for any $\lambda \in \lambda_{0}+t_{f} \mathbb{B}_{\Lambda}, \mu \in \mu_{0}+t_{\Phi} \mathbb{B}_{M}$,

$$
\begin{aligned}
e(W V(\lambda, \mu), W V) \leq & m_{f} h_{\Phi}\left\|\mu-\mu_{0}\right\|^{\alpha}+n_{f}\left\|\lambda-\lambda_{0}\right\|^{\delta}+\left(\frac{2 m_{f}^{2} h_{\Phi}}{l_{s}}\right)^{\frac{1}{\beta}}\left\|\mu-\mu_{0}\right\|^{\frac{\alpha}{\beta}} \\
& +\left(\frac{2 m_{f} n_{f}}{l_{s}}\right)^{\frac{1}{\beta}}\left\|\lambda-\lambda_{0}\right\|^{\frac{\delta}{\beta}}
\end{aligned}
$$

Moreover, if $\beta=1$, then

$$
e(W V(\lambda, \mu), W V) \leq \frac{\left(2 m_{f}+l_{s}\right) m_{f} h_{\Phi}}{l_{s}}\left\|\mu-\mu_{0}\right\|^{\alpha}+\frac{\left(2 m_{f}+l_{s}\right) n_{f}}{l_{s}}\left\|\lambda-\lambda_{0}\right\|^{\delta}
$$

Proof The proof is similar to Theorem 4.1 and is omitted here. 
Authors' contributions

The authors contributed equally and significantly in writing this paper. All authors read and approved the final manuscript.

\section{Author details}

${ }^{1}$ School of Electrical Engineering, Chongqing City Management Vocational College, Chongqing, 401331, China. ${ }^{2}$ College of Mathematics and Statistics, Nanyang Normal University, Nanyang, Henan 473061, China.

\section{Acknowledgements}

Xian-Fu Hu would like to thank Dr. Xue for his hospitality during his stay from July 2016 to August 2016 at College of Mathematics and Statistics of the Nanyang Normal University in China.

Received: 23 September 2016 Accepted: 8 November 2016 Published online: 29 November 2016

\section{References}

1. Li, XB, Li, SJ: Höder continuity of perturbed solution set for convex optimization problems. Appl. Math. Comput. 232, 908-918 (2014)

2. Yen, ND: Hölder continuity of solutions to parametric variational inequalities. Appl. Math. Optim. 31, 245-255 (1995)

3. Mansour, MA, Riahi, H: Sensitivity analysis for abstract equilibrium problems. J. Math. Anal. Appl. 306, 684-691 (2005)

4. Anh, LQ, Khanh, PQ: On the Hölder continuity of solutions to multivalued vector equilibrium problems. J. Math. Anal. Appl. 321, 308-315 (2006)

5. Chen, CR: Hölder continuity of the unique solution to parametric vector quasiequilibrium problems via nonlinear scalarization. Positivity 17, 133-150 (2013)

6. Li, SJ, Li, XB: Hölder continuity of solutions to parametric weak generalized Ky Fan inequality. J. Optim. Theory Appl. $149,540-553(2011)$

7. Li, XB, Long, XJ, Zeng, J: Hölder continuity of the solution set of the Ky Fan inequality. J. Optim. Theory Appl. 158, 397-409 (2013)

8. Wangkeeree, R, Preechasilp, P: On the Hölder continuity of solution maps to parametric generalized vector quasi-equilibrium problems via nonlinear scalarization. J. Inequal. Appl. (2014). doi:10.1186/1029-242X-2014-425

9. Peng, $Z Y$, Yang, $X M$, Teo, KL: On the Hölder continuity of approximate solution mappings to parametric weak generalized Ky Fan inequality. J. Ind. Manag. Optim. 11, 549-562 (2015)

10. Bonnans, JF, Shapiro, A: Perturbation Analysis of Optimization Problems. Springer, New York (2000)

11. Bednarczuk, EM: Weak sharp efficiency and growth condition for vector-valued functions with applications. Optimization 53, 455-475 (2004)

12. Luc, DT: Theory of Vector Optimization. Lecture Notes in Economics and Mathematical Systems. Springer, Berlin-Heidelberg (1989)

\section{Submit your manuscript to a SpringerOpen ${ }^{\circ}$ journal and benefit from:}

- Convenient online submission

- Rigorous peer review

- Immediate publication on acceptance

- Open access: articles freely available online

- High visibility within the field

- Retaining the copyright to your article 